\title{
Image Sensing Scheme Enabling Fully-Programmable Light Adaptation and Tone Mapping with a Single Exposure
}

\author{
Jorge Fernández-Berni, Fernanda D. V. R. Oliveira, Ricardo Carmona-Galán, Member, IEEE, \\ and Ángel Rodríguez-Vázquez, Fellow, IEEE
}

\begin{abstract}
This letter presents new insights into a High Dynamic Range (HDR) technique recently reported. We demonstrate that two intertwined photo-diodes per pixel can perform tone mapping under unconstrained illumination conditions with a single exposure. Experimental results attained from a prototype chip confirm the proposed theoretical framework. It opens the door to the realization of imagers providing HDR images free of artifacts without requiring any digital post-processing at all.
\end{abstract}

Index Terms-high dynamic range, split-diode, automatic adaptation, single exposure, tone mapping.

\section{INTRODUCTION}

$\mathbf{T}$ HERE are application frameworks in artificial vision characterized by totally unstructured illumination environments, e.g. automotive imaging. Traditional HDR techniques [1] are not suitable in such environments. They give rise to artifacts caused by motion, light flicker, etc. during the cumbersome process of rendering the final image. In order to reach the required performance in those scenarios, industry is departing from standard solutions where reducing the pixel pitch is the primary design driver. A representative example is the split-diode HDR technique recently reported [2]. Two photodiodes are included per pixel for overlapping exposures. Although the final image representation must still be generated after digitization, this technique mitigates undesired artifacts and distortion when compared to other approaches.

We recently proposed a two-photodiode HDR technique as well [3]. A major asset of our technique is that, unlike in [2], tone mapping is inherent to the adaptation process. Another advantage is that a single exposure suffices. On the flip side, some extra transistors are needed with respect to the pixel in [2] and global shutter is mandatory. In this letter, we describe how the technique introduced in [3] can be further simplified. More importantly, we mathematically demonstrate that the resulting simplified sensing scheme can accommodate any possible illumination conditions. Experimental results endorsing the underlying theoretical framework are presented.

J. Fernández-Berni, R. Carmona-Galán and Á. Rodríguez-Vázquez are with the Instituto de Microelectrónica de Sevilla (IMSE-CNM), CSIC-Universidad de Sevilla, 41092 Seville, Spain (contact email: berni@imse-cnm.csic.es).

F. D. V. R. Oliveira is with Universidade Federal do Rio de Janeiro, 21941901 Rio de Janeiro, Brazil (contact email: fernanda.dvro@poli.ufrj.br).

This work has been partially funded by the Spanish Government through project TEC2015-66878-C3-1-R MINECO (co-funded by ERDF-FEDER), Junta de Andalucía through project TIC 2338-2013 CEICE, CNPq/Brazil through research grant 204382/2014-9 and the Office of Naval Research (USA) through grant N000141410355.

Manuscript received XXX XX, XXXX; revised XXX XX, XXXX.

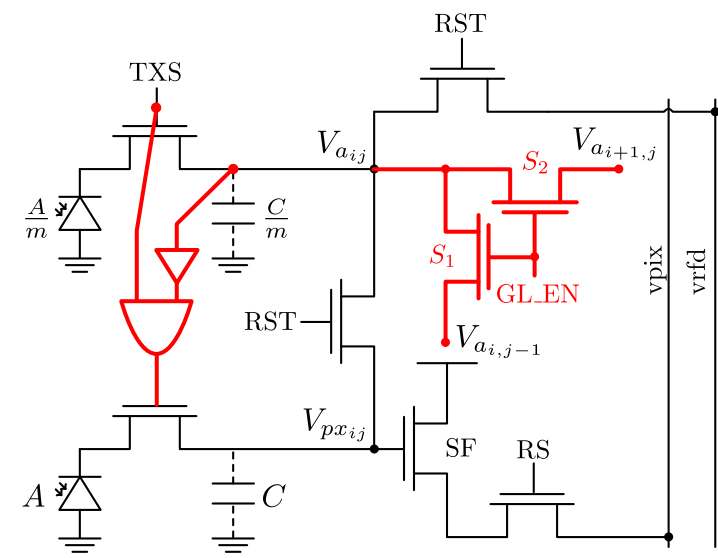

Fig. 1. Circuitry at pixel level for the proposed sensing scheme.

\section{Fully-Programmable Light AdAPtation}

The pixel to be considered is the same as in [3]. The extra elements required with respect to the pixel in [2] are depicted with a thicker red line in Fig 1. The image capture stage is governed by three temporal parameters: a reset period $T_{R S T}$, a maximum photo-integration period $T_{\max }$ and a period $T_{S}$ where the ambient illumination is sensed. An illustrative timing diagram is depicted in Fig. 2. The labels max and min refer to those pixels where the maximum and minimum photocurrents, respectively, are generated. The rest of pixels would evolve along curves in between. The parameter $T_{S}$ constitutes a simplification with respect to the multiple samplings of the mean illumination proposed in [3]. We prove next that this simplification permits programmable adaptation to any possible illumination conditions. The switches $S_{1,2}$ enable charge redistribution while the global signal GL_EN is asserted. This forces all the voltages $V_{a_{i j}}$ to follow the same evolution:

$$
V_{a_{i j}}(t)=V_{r s t}-\frac{\sum_{\forall i, j} I_{p h_{i j}} / m}{M \times N \times C / m} t \quad \forall t \in\left[0, T_{S}\right)
$$

where $M \times N$ is the image resolution, $C$ is the sensing capacitance and $m$, equal to 1 in our prototype, is an area scale factor as described in [3]. Note that $V_{r s t}=$ vfrd if hard reset is applied. We can define $\bar{I}_{p h}=\sum_{\forall i, j} I_{p h_{i j}} /(M \times N)$ as the average photo-current across the image, directly proportional to the mean illumination impinging on the sensor. Once GL_EN is switched low, we have that: 


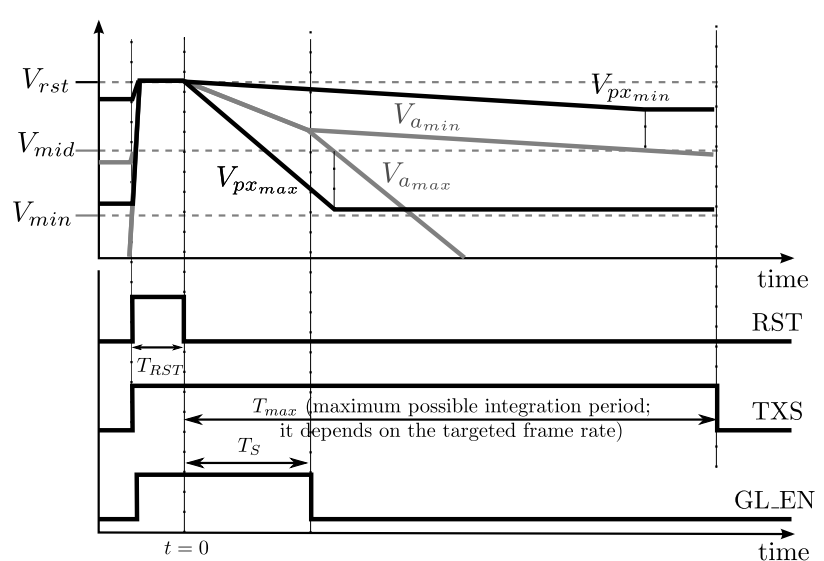

Fig. 2. Timing diagram corresponding to an image capture.

$$
V_{a_{i j}}(t)=V_{r s t}-\frac{\bar{I}_{p h}}{C} T_{S}-\frac{I_{p h_{i j}}}{C}\left(t-T_{S}\right) \quad \forall t \geq T_{S}
$$

Let us assume now that $T_{S}$ is chosen in such a way that all the voltages $V_{a_{i j}}$ cross the middle value of the signal range, denoted as $V_{\text {mid }}$, at some instant $t=T_{i j}>T_{S}$. The input threshold voltage of the digital buffer connected to $V_{a_{i j}}$ is designed to coincide with $V_{\text {mid }}$ [3]. The photo-integration interval defining the pixel value thus extends until $t=T_{i j}$, being $V_{p x_{i j}}\left(T_{i j}\right)=V_{r s t}-I_{p h} T_{i j} / C$. This interval can be mathematically defined by simply setting $V_{a_{i j}}\left(T_{i j}\right)=V_{m i d}$ in Eq. 2 and solving for $T_{i j}$. After some algebra, the following linear expression for $V_{p x_{i j}}$ can be easily obtained:

$$
V_{p x_{i j}}\left(T_{i j}\right)=V_{m i d}+\frac{\bar{I}_{p h}-I_{p h_{i j}}}{C} T_{S}
$$

A first aspect to be highlighted from Eq. 3 is that the mid point of the available signal range is matched with the average illumination impinging on the sensor; i.e. $I_{p h_{i j}}=\bar{I}_{p h}$ always leads to $V_{p x_{i j}}=V_{\text {mid }}$, no matter the specific value of $\bar{I}_{p h}$ considered. As a result, any average illumination level can theoretically be accommodated. The minimum representable photo-current, $I_{p h_{m i n}}$, is fundamentally limited by the noise floor and $T_{\max }$, as in any other sensing scheme based on photo-charge integration. Leaving aside noise, for $T_{\max } \rightarrow \infty$, $I_{p h_{\text {min }}} \rightarrow 0$, no matter the value of $T_{S}$ chosen. Concerning the maximum representable photo-current, $I_{p h_{\max }}$, we simply have to set $V_{p x_{i j}}\left(T_{i j}\right)=V_{\min }$ in Eq. 3 and solve for $I_{p h_{i j}}$ :

$$
I_{p h_{\max }}=\bar{I}_{p h}+\frac{C\left(V_{r s t}-V_{\min }\right)}{2 T_{S}}
$$

which means that any possible maximum photo-current can theoretically be linearly accommodated within the signal range by properly adjusting $T_{S}$. For $T_{S} \rightarrow 0, I_{p h_{\max }} \rightarrow \infty$. Our sensing scheme does not therefore impose any theoretical limits for implementing linear tone mapping according to specific illumination conditions. Practical limitations will do arise from system-level requirements-frame rate-setting a limit for $T_{\max }$ and from the minimum interval $T_{S}$ achievable by a particular physical realization. This scheme also introduces an

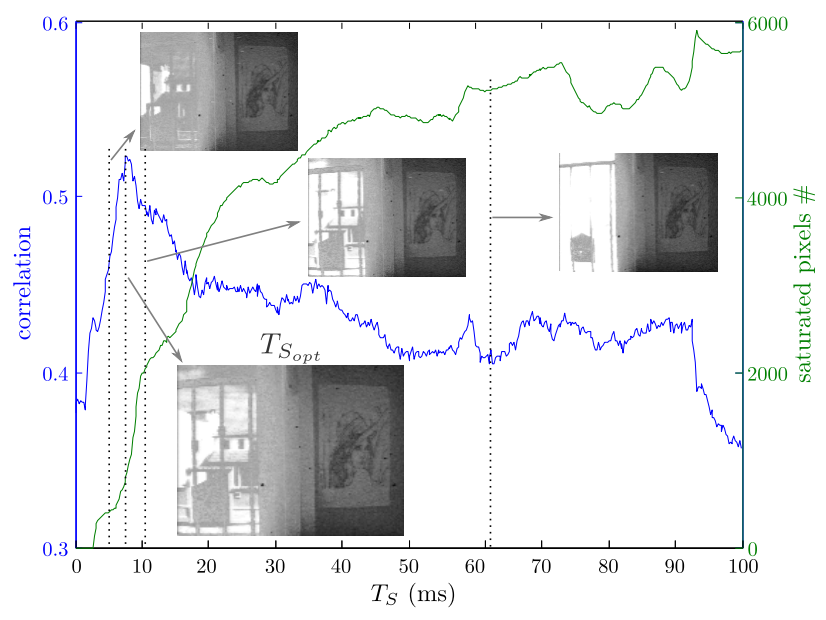

Fig. 3. Experimental results from a prototype chip.

additional source of spatial noise arising from process-induced variations of $T_{i j}$-e.g. due to mismatch in $V_{\text {mid }}$. This noise can be expressed as a term added to the Photo-Response NonUniformity (PRNU).

\section{EXPERIMENTAL RESULTS}

The same prototype and benchmark - based on correlation with a perfectly equalized histogram—as in [3] are used for the tests. Assuming that $I_{p h_{m i n}}$ is determined by prescribed values of $T_{\max }$ and noise floor, our tests focus on Eq. 4. This equation states that the smaller the value of $T_{S}$, the wider the range of illumination tones linearly mapped into the available signal swing according to Eq. 3. For a particular scene featuring certain levels of illumination, there must be an optimum value $T_{S_{\text {opt }}}$ rendering the best tone mapping of those levels in terms of the aforementioned benchmark. For $T_{S}<T_{S_{\text {opt }}}$, we would be wasting part of the signal swing allocated for high levels of illumination that do not actually exist in the impinging radiation. Conversely, for $T_{S}>T_{S_{\text {opt }}}$, the number of saturated pixels would increase associated with tones that do impinge on the sensor. This is exactly the behavior we find in the tests performed. An example is shown in Fig. 3 for a HDR scene containing both sunlight and indoor levels of illumination. $T_{S}$ is swept from $200 \mathrm{~ns}$ up to $100 \mathrm{~ms}$ with steps of $200 \mu \mathrm{s}$. For each value of $T_{S}$, we compute the number of saturated pixels as well as the correlation of the resulting image histogram with the ideal equalized histogram.

\section{CONCLUSIONS}

An image sensing scheme capable of linearly adapting to any possible illumination conditions with a single exposure has been described and experimentally proved.

\section{REFERENCES}

[1] A. Spivak et al., "Wide-dynamic-range CMOS image sensorscomparative performance analysis," IEEE Trans. Electron Devices, vol. 56, no. 11, pp. 2446-2461, 2009.

[2] T. Willassen et al., "A $1280 \times 10804.2 \mu \mathrm{m}$ split-diode pixel HDR sensor in 110nm BSI CMOS process," in Int. Image Sensor Workshop, 2015.

[3] J. Fernández-Berni et al., "Single-exposure HDR technique based on tunable balance between local and global adaptation," IEEE Trans. Circuits Syst. II, 2015, DOI 10.1109/TCSII.2015.2505263. 